\title{
Further studies on the retrocorneal membrane-endothelium relationship
}

\author{
EMIL S. SHERRARD \\ Pocklington Eye Transplantation Research Unit, Royal College of Surgeons of England, London
}

Partial penetrating keratoplasty may fail for a number of reasons. By improved surgical technique, instrumentation, therapy, and methods of managing donor material some of $\omega$ these reasons are suppressed, thereby increasing the relative importance of the remainder. $\vec{N}$ Hence post-graft membranes (PGMs) have come to occupy a high place in the pathology $\stackrel{\infty}{\infty}^{\infty}$ of corneal grafts (Rycroft, I965; Brown and Kitano, I966; Kurz and D’Amico, I968).

Post-graft membrane is the name applied to errant fibrous tissue which often develops after full-thickness keratoplasty from the host/graft junction and forms a sheet of variable dimensions and density behind the graft (Werb, 1962). Similar structures which occur after penetration of the posterior corneal layers by mechanical injury other than grafting, and by degenerative processes have been called post-trauma membranes (PTM) and postdisease membranes (PDM) respectively (Sherrard and Rycroft, 1967a). The three aetiological types are grouped under the heading retrocorneal membrane (RGM). Other retrocorneal structures can be classified on anatomical criteria. There are those comprising iris tissue, fibrin, epithelium, cell and blood debris, or a combination of them. The membranes which are under investigation here are those composed of corneal stromal elements. Occasionally they are enhanced by anterior iris synechia but this is to be regarded as a secondary feature.

In earlier reports (Sherrard and Rycroft, $1967 \mathrm{a}, \mathrm{b}$ ), it was suggested that the corneal endothelium prevents and does not augment PGM growth, and it was mentioned that total PGMs result after the complete removal of the graft endothelium in rabbits. Since that time one hundred further experiments, involving destruction of the corneal endothelium adjacent to retrocorneal wounds in rabbits, have been carried out. Basically the results 3 agree with those of similar investigations by Brown and Kitano (1966), and have not, $\stackrel{8}{\circ}$ therefore, been published. However, they greatly strengthen the view that RGMs will develop only in the absence of functional endothelium. Figs $I$ and 2 illustrate PGM $\frac{}{5}$ formation after partial (Fig. I) and total (Fig. 2) removal of the graft endothelium.

The RGM-endothelium relationship has been investigated under more closely controlled conditions. Small fragments of corneal stroma were explanted into the anterior chamber. The explants represented potential RCMs and were either placed directly against healthy endothelium or totally separated from it. They were all of approximately the same size to standardize the mass of potential RGM and all were placed well away from the iris and penetrating corneal wounds to avoid their involvement in iris synechia, foreign body (suture) reaction, vascularization, oedema, epithelialization, and contamination by any? PTM issuing from a large wound. Initially only autoexplants were prepared to evade immunological complications.

The results demonstrate that the integrity of the endothelium is essential to the prevention of retrocorneal fibrosis of stromal origin. 
(1)

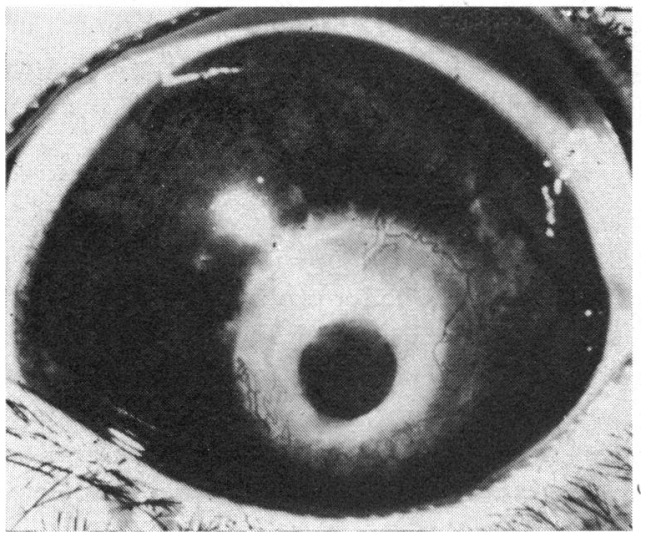

(2)

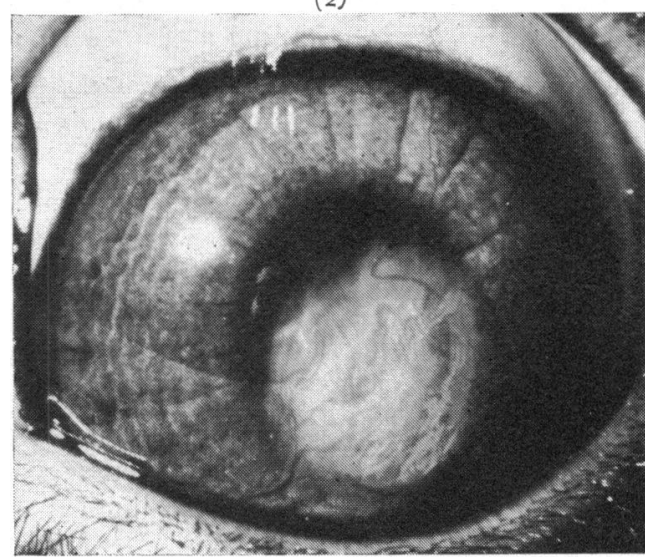

FIG. I Penetrating 6. I $\mathrm{mm}$. autograft. 90 days postoperatively. The marginal endothelium of the graft was removed to a distance of about $2 \mathrm{~mm}$.

NOTE: PGM extends from the host/graft junction to a central island of remaining endothelium. Blood vessels lie in clear graft anterior to PGM. (From a colour transparency).

FIG. 2 Penetrating 6. I $\mathrm{mm}$. homograft. I year postoperatively. The entire endothelium of the graft was removed.

Note: Thick total PGM. The blood vessels lie in clear graft anterior to PGM. (From a colour transparency).

Observations of the clinical progress and histological preparations of these experiments are recorded, and forthcoming explanations for the characteristic retrograft position of the PGM and for the great dimensional variation of RGMs in general are discussed.

\section{Material}

The left eyes of healthy adult rabbits of many breeds were used, but albino forms were avoided to facilitate clinical observations.

Halothane anaesthesia (Sherrard, 1966) was used for all operations. The animals were heparinized ( 1,000 units intravenously) immediately before surgery to prevent clotting of the aqueous humour. Wounds were closed with $8 \cdot 0$ virgin silk interrupted and/or continuous sutures, which were removed 9 to 14 days postoperatively under local anaesthesia ("Ophthaine"), or with $10 \cdot 0$ Perlon which was left in situ. Fixed explants were attached to the posterior corneal surface by a single 10.0 Perlon suture. Chloromycetin ointment was instilled daily into the operated eyes for 2 weeks. Most of the animals were killed by air embolism 21 days postoperatively but a few were left for 2 months and longer and others were killed at 2 weeks. Histological studies were made from $5 \mu$ to $8 \mu$ paraffin and ester wax sections of Bouin fixed corneae stained with Masson's trichrome, haematoxylin and eosin, or periodic acid-Schiff (PAS).

\section{Method}

Three variations of stromal autoexplants were performed and these followed by a series of stromal homoexplants and some anterior chamber implants which acted as controls. The autoexplants consisted of fragments of stroma removed from the host eye.

\section{(I) Free explants: 4 eyes}

The epithelium was removed from the inferonasal quadrant of the cornea of the anaesthetized rabbit by repeated scraping with a scalpel, washing with jets of saline, and wiping with a soft sponge. A square of the exposed stroma about $2 \times 2 \times 0.2 \mathrm{~mm}$. was then cut from the centre of the quad- 
rant and inserted into the anterior chamber through a very small superior limbal incision. Little or no aqueous humour was lost and reformation of the anterior chamber was unnecessary. This technique was unsatisfactory owing to the involvement of the iris.

(2) Fixed explants: 30 eyes

(a) The explants were prepared as in ( $\mathrm{I}$ ). A large penetrating incision about $1 \mathrm{~mm}$. from the limbus and extending from 9 to 3 o'clock was made and the superior half of the cornea reflexed upon the inferior. The explant was then fixed centrally to the retrocorneal surface with a Perlon suture. The fold in the cornea facilitated placing the suture which, as far as was possible, was passed through the Descemet's and endothelial layers only. The wound was closed and the anterior chamber reformed with air by means of a cannula passed through the wound. The same instrument served also to arrange the explant against the posterior corneal surface. In this way a potential RCM was placed well away from the iris and the penetrating incision and their associated complications.

(b) Five stromal fragments with their accompanying epithelium were transplanted as in (a) so that the epithelium lay against the endothelium.

(c) Five epithelio-stromal explants were placed so that the epithelium faced away from the host cornea.

\section{(3) Fixed explants separated from the host endothelium: 20 eyes}

These were prepared and fixed to the retrocorneal surface as in 2 (a), but a $6 \cdot \mathrm{I} \mathrm{mm}$. disc of 0.003 in. thick silicone rubber was interposed between each explant and cornea. A single Perlon suture served to retain both the explant and the implant in position.

\section{(4) Free silicone implants: 18 eyes}

$6 \cdot 1 \mathrm{~mm}$. silicone discs were sutured centrally to lengths of fine silicone rubber tubing. This had been previously tailored to fit into the anterior chambers of recipient eyes in such a way that each would extend across the transverse diameter and follow the inner contour of the cornea. One end of each length of tubing was wedged into the angle at the 9 o'clock position and the other at 3 o'clock. The tubing thus acted as a weak spring which gently pressed the silicone disc against the back of the central area of the cornea. In this way contact between the silicone disc and the posterior corneal surface was retained without the direct use of sutures and the resulting perforations in the deepest corneal layers. Four of the implants moved superiorly and became contaminated by PTM growing from the large insertion incision. The remainder succeeded well.

\section{(5) Fixed implants: 6 eyes}

Silicone rubber discs as used in (3) without stromal explants were sutured to the retrocorneal surface.

Experiments (2) and (3) above were repeated using stromal fragments from another rabbit. i.e. stromal homoexplants.

Unfortunately it was not possible to preserve silicone rubber in wax sections, but as it became dislodged during flattening on warm water a clearly identifiable impression of it was left in the final preparations.

\section{Results}

The free stromal autoexplants (Method I) all sank in the anterior chamber. Two came to rest in the angle, one on the iris, and the other wedged between iris and cornea $2 \mathrm{~mm}$. from the limbus. No evidence of growth was observed, although in sections each appeared to consist of normal healthy stromal tissue. Those in contact with the cornea were surrounded by endothelium which exhibited typical kidney-shaped nuclei close to the cornea but gave way to pigmented cells which were continuous with those of the iris. The explant which contacted the iris alone was engulfed by its pigmented tissue. 
Two features were noteworthy in these results. None of the explants showed signs of growth, which can probably be related to the insignificant disturbance caused to the anterior chamber, and hence to the endothelium, and all were completely endothelialized by corneal and/or iris cells.

To prevent explants contacting the iris they were fixed to the posterior corneal surface (Method 2). All were observed clinically to round off between the $4^{\text {th }}$ and 8 th postoperative days (Fig. 3) and to show no changes in size or shape thereafter, although they generally became less and less distinct. Sections revealed that the explants resembled normal stroma except for disorganization of the lamellae (Fig. 4). They were undoubtedly viable. Transplants without epithelium (Method 2a) lay immediately against Descemet's membrane, there being no visible endothelium nor neofibrous tissue between them. The part of the explants originally exposed to the aqueous humour was now covered by a layer of neofibrous tissue which extended a short distance beyond the transplant onto Descemet's membrane. Its thin fibres lay parallel to the curvature of the cornea and it was lined posteriorly by a single layer of endothelium which was continuous with that of the cornea. In some cases a very thin secondary Descemet's membrane between the neofibrous tissue

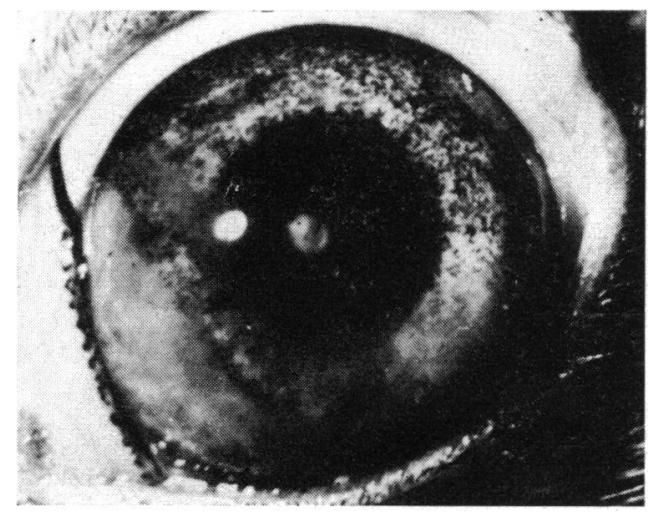

FIG. 3 Stromal autoexplant fixed directly to retrocorneal surface. 2 I days postoperatively.

NOTE: The explant which was square and flat has become shrunken and globular. (From a colour transparency).

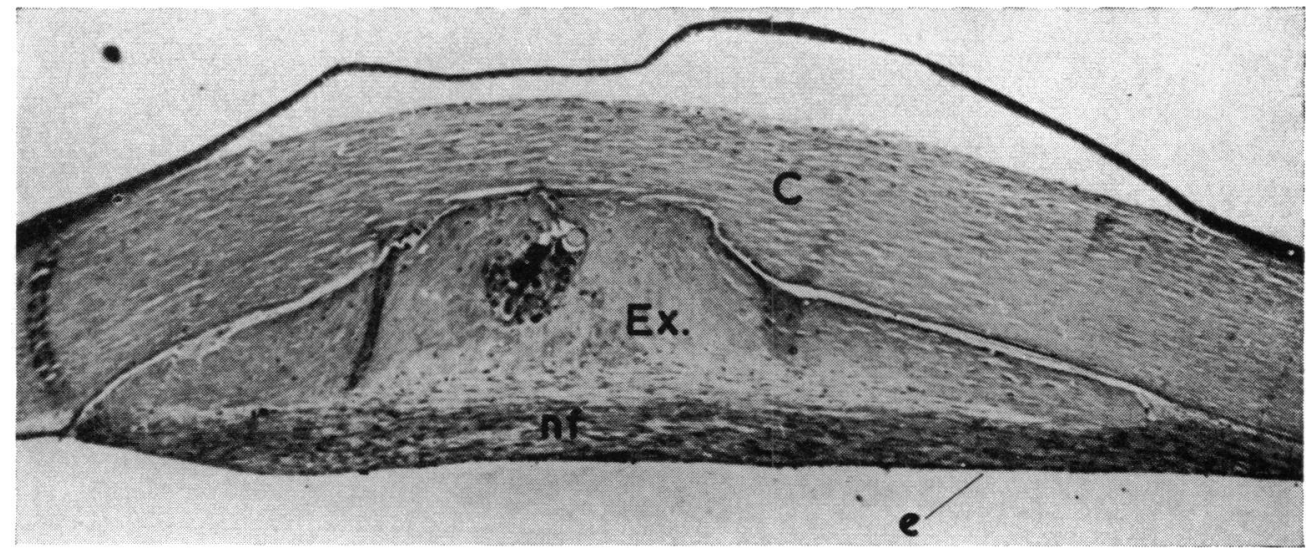

FIG. 4 Section through stromal autoexplant fixed directly to retrocorneal surface. 2 I days postoperatively.

NOTE: Neofibrous tissue (dark) has developed on back of explant. Masson's trichrome. $\times 5^{0}$. C-cornea; Ex-explant; nf-neofibrous tissue; e-endothelium. 
and the endothelium was demonstrable with PAS staining. One specimen was sectioned $\frac{0}{3}$ I year and I month postoperatively, after the natural death of the animal. Clinically the explant was just discernible as a whispy blemish in the cornea (Fig. 5). Histologically it was almost indistinguishable from the corneal stroma into which it had become embedded.o There was a thick secondary Descemet's membrane, and a complete endothelium posteri- $\frac{\bar{D}}{\overline{\bar{N}}}$ orly. Specimens which included epithelium on the corneal side of the stromal transplant $\frac{\tilde{\sigma}}{\widetilde{\sigma}}$ (Method 2b) (Fig. 6) were similar in all respects to the pure stromal explants except that $\frac{}{0}$ epithelium lay between Descemet's membrane and the explanted stroma, or formed ${ }^{\infty}$ islands of cells embedded in the latter. In some cases the fixing suture holes in both? cornea and explant were epithelialized, demonstrating some regeneration of the explanted $\vec{\omega}$ epithelium.

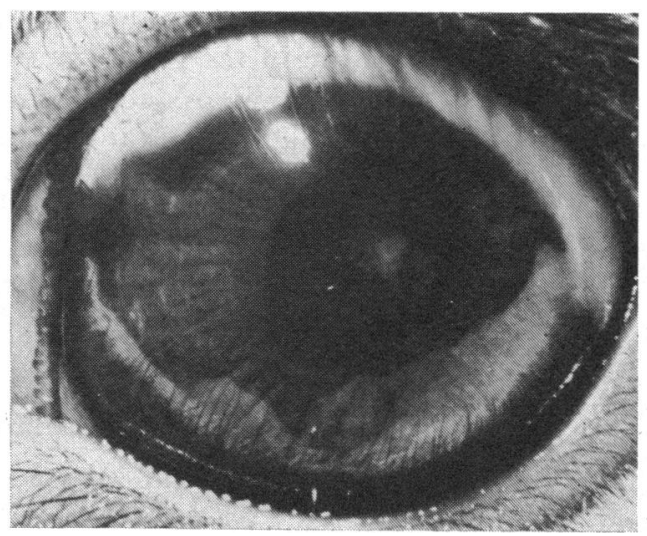

FIG. 5 Stromal autoexplant I year and I month postoperatively.

NOTE: Explant has become diffuse and indistinct. (From a colour transparency).

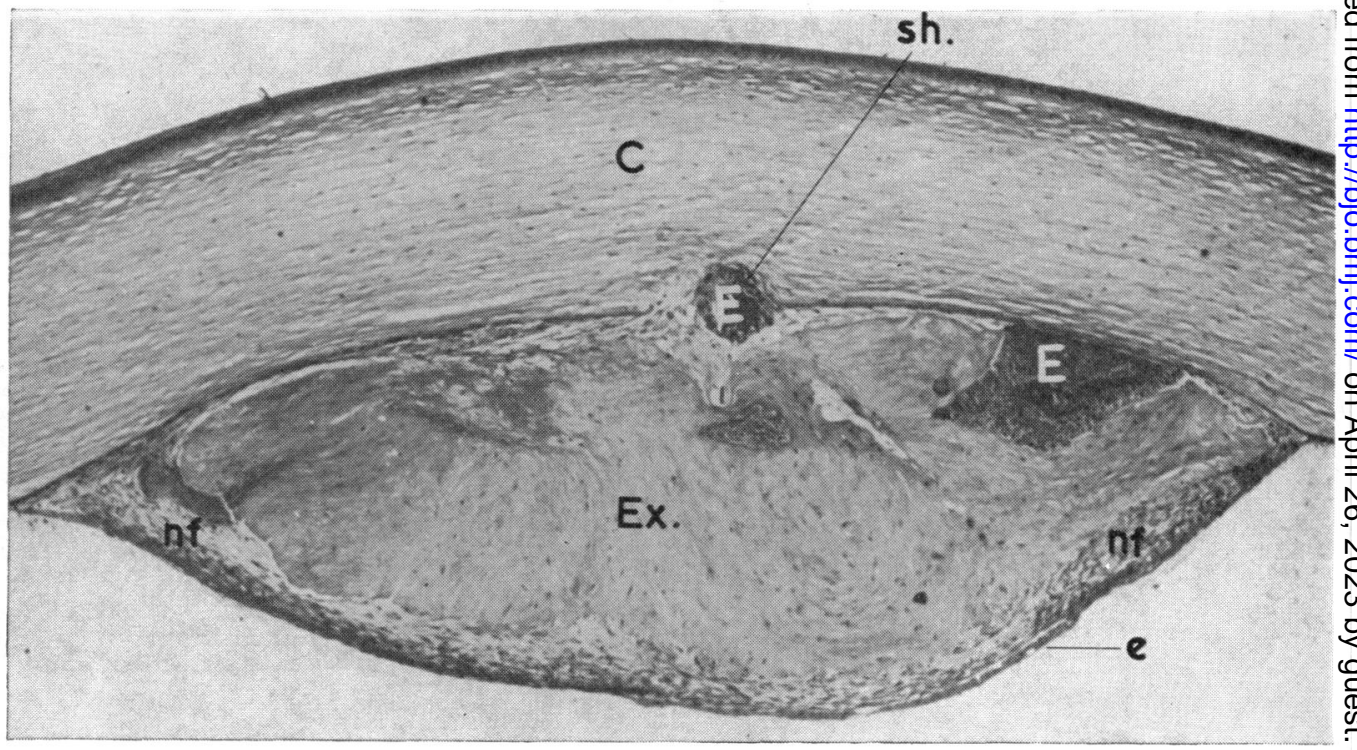

FIG. 6 Section through epitheliostromal autoexplant. 2 I days postoperatively.

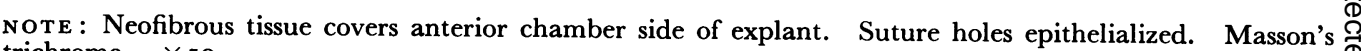
trichrome. $\times 50$

C-cornea; Ex-explant; nf-neofibrous tissue; E-epithelium; e-endothelium; sh-suture hole. 
Explants with the epithelium on the anterior chamber side (Method 2c) were accompanied by neofibrous tissue but this never extended behind the epithelium. Endothelialization likewise stopped abruptly where it met epithelium. Loose spherical cells betrayed epithelial regeneration; the stratified arrangement was lost.

It appears from these results that neofibrous tissue was regenerated from the explanted stroma but only where it was exposed to aqueous humour, for where it was covered by a layer of epithelium and, later, endothelium, no fibrosis occurred. Furthermore, endothelialization was limited to the fibroblastic tissue; it did not occur posterior to the transplanted epithelium.

The techniques employed in these experiments disturbed the anterior chamber, and therefore the endothelium, to a considerable degree, and undoubtedly retarded endothelialization of the explants, thus permitting some small growth. Consequently, it was felt that similar explants would grow freely if separated from the endothelium. Separation was achieved to a limited but sufficient extent by interposing a disc of silicone rubber between pure stromal explants and the retrocorneal surface (Method 3). Retardation of endothelialization by the removal of cells proved too traumatic and rendered the cornea opaque, preventing clinical observations, whereas the silicone disc simulated a Dohlman membrane (Dohlman, Brown, and Martola, 1967) and allowed daily examination of the transplanted fragments of stroma.

The explants did not round off but grew rapidly in several directions, in contrast to those of previous experiments with no silicone partition. "Usually growth did not occur from all sides of the explants, but was always strictly limited to the silicone disc. Typical results are shown in Figs 7 and 8, and comparison with Fig. 3 indicates the extent of the growth which has occurred, since the original transplanted fragments of stroma were approximately the same size in each case.

(7)

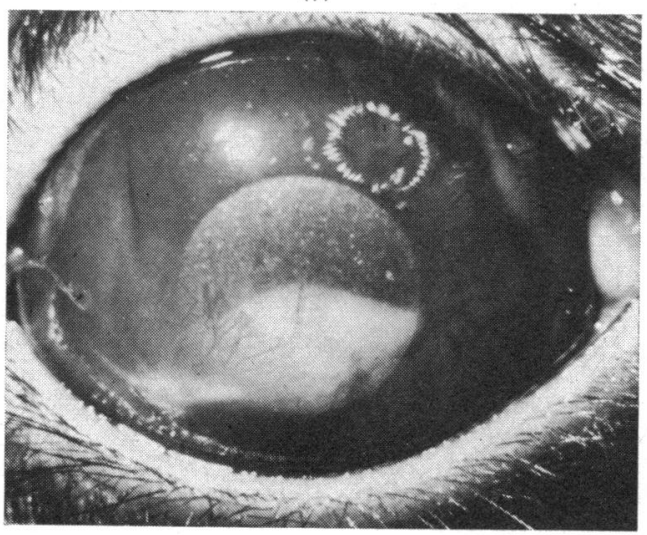

(8)

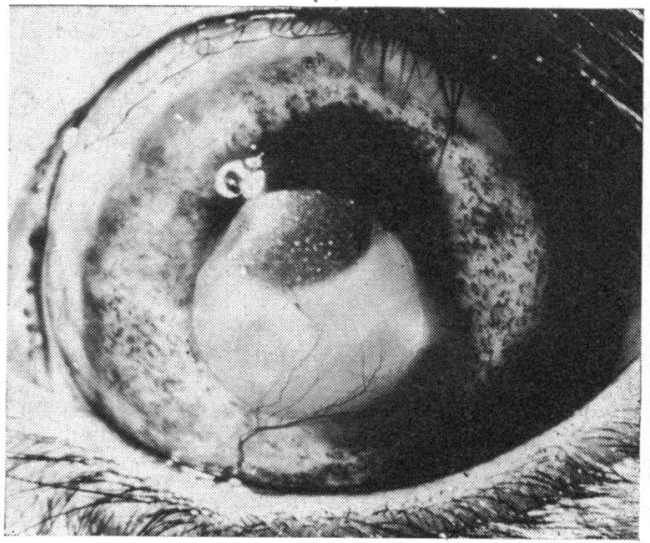

FIG. 7 Stromal autoexplant posterior to silicone rubber disc. 2 I days postoperatively.

NOTE: Explant has "grown" to cover most of inferior half of silicone disc, and stops abruptly as its edge. (From a colour transparency).

FIG. 8 Stromal autoexplant posterior to silicone rubber disc. 90 days postoperatively.

NOTE : Explant has "grown" to cover most of disc, and stops abruptly at its edge. This stage was reached by $15^{\text {th }}$ day. (From a colour transparency). 
In some of the specimens the silicone disc did not lie in total contact with the retrocorneal surface and aqueous humour penetrated between the two. In these cases, although the corneae became very hazy, fibrous tissue was seen to form in the space. Histologically it appeared as true PTM arising from the host corneal stroma and passing through the suture holes into the anterior chamber. It never occurred where the silicone-corneal seal was complete.

All the corneae became slightly vascularized from the limbus.

The increase in size of the explants was seen in sections to be due to the formation of considerable amounts of neofibrous tissue which apparently originated from the trans- ? planted stroma, since the two merged together. It spread outwards across the posterior $\vec{\omega}$ face of the silicone disc and just overflowed its edge to contact the corneal endothelium (Fig. 9) or PTM if present. The original explant was relatively unchanged and retained the basic structure and staining properties of normal stroma. The neofibrous tissue, however, was more cellular and of variable density. Distally it was lined posteriorly by $\stackrel{\sim}{\infty}$ endothelium and, in some cases, a thin secondary Descemet's layer. Here it resembled a mature RCM (Sherrard and Rycroft, 1967a). Proximally endothelium was absent and the structure resembled a juvenile RCM.

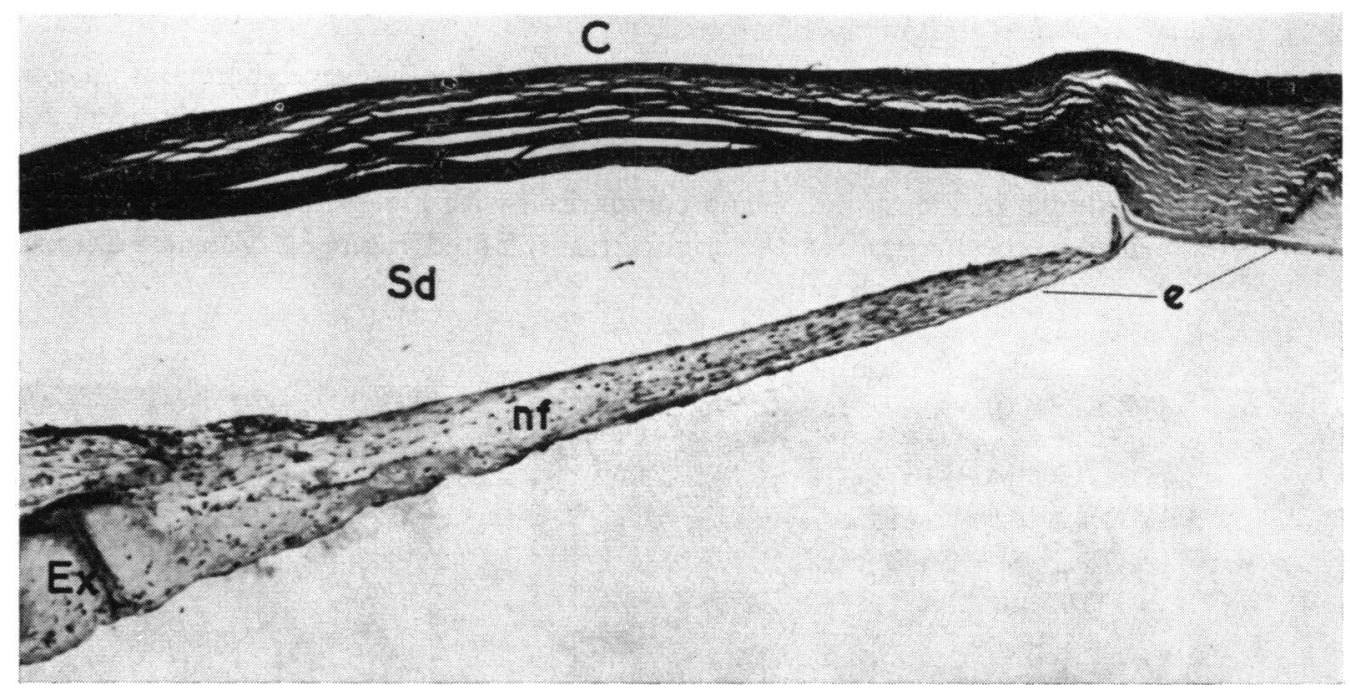

FIG. 9 Section through stromal autoexplant posterior to silicone rubber disc. 2 I days postoperatively.

NOTE: The space previously occupied by the silicone is enlarged by artefact, but the shape of its angular extremity is clearly seen (right). The neofibrous tissue contacts corneal endothelium at the edge of the disc. Masson's trichrome. $\times 5^{\circ}$

C-cornea; Ex-explant; Sd-space previously occupied by silicone disc; $\mathrm{nf}$-neofibrous tissue; e-endo- $N$ thelium.

The endothelium was destroyed anteriorly to the silicone disc but was normal immediately beyond its periphery in all the specimens.

The noteworthy features of these results were:

(i) The extensive apparent growth of the explants on the endothelium-free silicone disc, and its abrupt cessation at the edge of the disc where the neofibrous tissue met and was overgrown by endothelium. This strongly indicates the necessity of endothelium in the prevention of retrocorneal fibrosis. 
(ii) The PTM issuing from the suture holes was likewise strictly limited to the circumference of the silicone disc, within which endothelium was absent but beyond which it was normal, and occurred only in specimens where the silicone-corneal junction was incomplete. Concurrently, in these specimens alone, the portion of cornea anterior to the silicone implant became very hazy. This suggested that access to aqueous humour is necessary to retrocorneal fibrosis and that such is normally prevented by the endothelium. A closefitting silicone membrane can apparently have the same effect.

It was possible that the neofibrous tissue associated with explants and posterior to the silicone disc had its origin in tissues other than in the transplanted stroma, e.g. the endothelium or PTM. Therefore, a series of control experiments was done without explants and also in which the conditions for PTM development were avoided. Silicone discs were maintained in contact with the retrocorneal surface without the use of direct sutures (Method 5) and thus without perforations in Descemet's membrane. No fibrosis anterior or posterior to the silicone occurred, demonstrating that the neoblastic tissue present in previous results was unlikely to have developed from a source other than the explanted stroma or PTM. The latter could not be overlooked for union between them and neofibrous tissue posterior to the silicone disc at its edge had been observed. Furthermore, it was conceivable that minute PTMs had been missed in the specimens in which none was thought to exist.

Silicone discs without accompanying explants were sutured to the retrocorneal surface (Method 4). PTMs grew from the suture holes in all eyes but one, which possessed the only completely flat implant, and were limited to the circumference and anterior face of the silicone.

It seems, therefore, that stromal explants separated from the corneal endothelium by silicone discs grow freely to produce connective tissue sheets which are comparable to true RGMs. It seems certain that retrocorneal fibrosis is prevented by the endothelium.

Because PGMs are a complication of keratoplasty in humans, and here usually of homografting, it was desirable to simulate the process by the use of homoexplants to discover whether or not they differed markedly from the autoexplants. Fragments of stroma from six rabbits were, therefore, transplanted to the retrocorneal surface of six others. The results differed from the autoexplants only in the total absence of retrocorneal neofibrosis, even though the anterior chamber had suffered the same degree of disturbance. The homoexplants were globular, apparently viable, and enveloped by normal endothelium (Fig. Io, overleaf).

Homoexplants separated from the cornea by silicone discs, clinically and histologically resembled autoexplants similarly placed, the only difference being that the homoexplants showed the first signs of growth 3 or 4 days later.

Insufficient homoexplants from which to draw conclusions have been observed, but the constancy of the few results suggests that growth of stromal fragments transplanted from one rabbit to the posterior corneal surface of another is inhibited even before endothelialization. This is possibly true also of full-thickness homografts.

\section{Comments}

It has been shown that fibrous tissue is generated from fragments of stroma transplanted into the anterior chamber in the same way as from stroma exposed to aqueous humour by trauma. The experimental use of such fragments has allowed greater control of the circumstances in which this generation occurs, particularly as regards the state of the asso- 


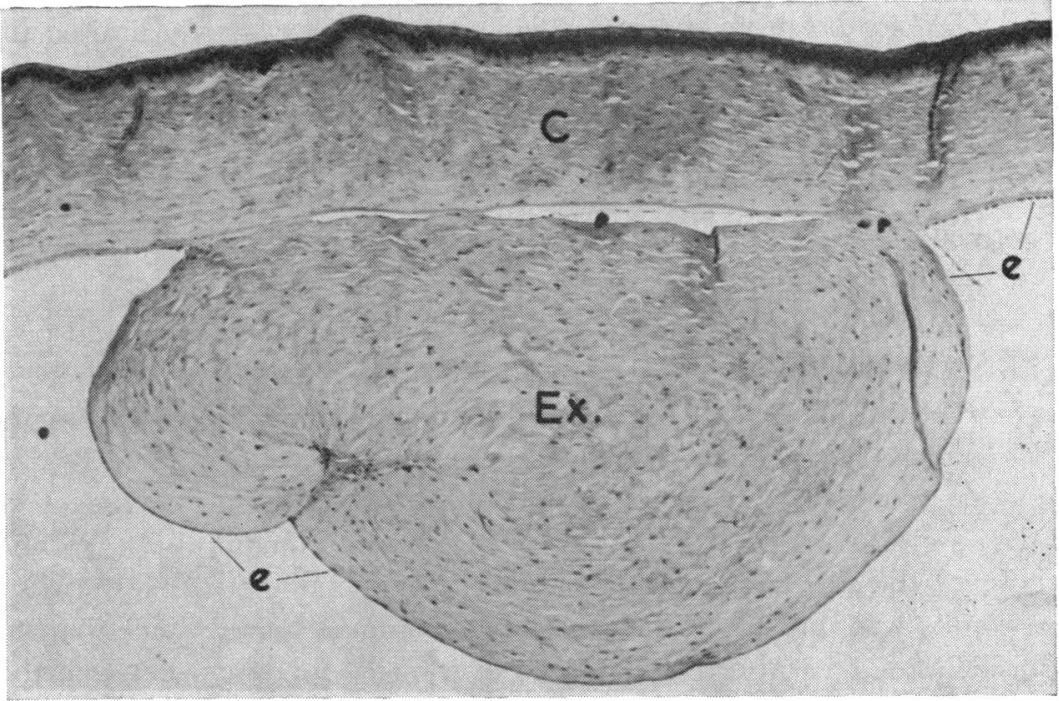

FIG. Io Section through stromal homoexplant fixed directly to retrocorneal surface. 28 days postoperatively.

Note: Total absence of neofibrous tissue. Complete endothelium. Good condition of explanted tissue. Masson's trichrome. $\times 5^{0}$

C-cornea; Ex-explant; e-endothelium.

ciated endothelium. Explants placed in contact with normal endothelium were quickly covered by it, and grew only slightly, whereas those separated from endothelium, and other ocular tissues, grew strongly until they met and were overgrown by endothelium. The method by which endothelium prevents retrocorneal fibrosis has not been investigated but, since silicone rubber apparently has the same effect, it is possible that the formation of a physical barrier to aqueous humour in the form of a secondary Descemet's membrane is important. However, these and previous experiments (Brown and Kitano, I966; Sherrard and Rycroft, $1967 \mathrm{~b}$ ) leave little doubt that endothelialization of posterior corneal wounds prevents the growth of RCMs.

Areas denuded of endothelium are rapidly recolonized (Chi, Teng, and Katzin, I960) and endothelium is never present anterior to established RGMs or stromal explants which contact the posterior corneal surface, and yet it covers them posteriorly. Possibly, therefore, multiplication of endothelial cells in the vicinity of retrocorneal fibrous tissue occurs essentially as a reparative phenomenon and not in response to a tactile stimulus as might appear from the endothelialization of explants and other foreign bodies (Thomas, 1955).

\section{Discussion}

A corneal injury which includes perforation of the Descemet's and endothelial layers normally potentiates two processes; multiplication of cells of the endothelium, which rarely occurs in normal adult corneae, and the development of a RGM from the stroma. Each process is preceded by a time lag. Amitosis in the endothelium occurs from five to seven hours after injury (Chi and others, 1960) and mitosis from 24 hours (Binder and Binder, 1957; Morton, Ormsby, and Basu, 1958; Chi and others, I96o; Mills and Donn, 196o; and others). A juvenile RCM appears on the $5^{\text {th }}$ post-injury day (Sherrard and Rycroft, 
1967a). The difference in "latent period" before each process ensures that a small, simple wound such as a retrocorneal scratch or uncomplicated piercing injury is endothelialized before fibroblasts flow out from the stroma onto the anterior chamber side of Descemet's membrane, with the result that healing is rapid and even. In the case of a large wound, including keratoplasty, complicated by some kind of damage to the adjacent endothelium, sufficient time may elapse for the formation and growth of a juvenile RGM before it meets healthy endothelium.

On consideration of the sequence of events which befall the average donated cornea from and including the death of the donor up to and including its relocation into the foreign environment of the acquired host, it seems not unreasonable to assume some functional deterioration of the endothelium (Peña-Carrillo and Polack, 1964; Robbins, Capella, and Kaufman, I965). The endothelium of the recipient cornea, on the other hand, is often in good condition at the time of grafting. The assumption is, therefore, that there is frequently a considerable difference between the functional ability of the host and graft endothelia, and that the latter may require longer to return to normality than the former, or indeed may fail to do so.

Providing now that the host cornea is sufficiently robust to receive and heal to the graft (Condition I of RCM development: Sherrard and Rycroft, I967b) and that the alignment of host and graft Descemet's membrane is not adequate (Condition 2 of RCM development), excess fibroblasts produced in the host stroma to repair the wound (Brown and Kitano, I966) will spill out into the anterior chamber to lay the foundations of a PGM. This will be arrested by endothelialization first from the host side, but free to grow behind the dormant endothelium of the graft until it recovers sufficiently to check the membrane. In this way PGMs occur, ranging in size from little more than enlarged scars at the hostgraft junction, when both host and graft endothelia are fully functional shortly after keratoplasty, to total retrograft sheets when the graft endothelium fails to recover or when the growing PGM does not contact it. Retardation or failure of recovery of the host endothelium results in the RGM growing back behind the host.

Wispy and digitate PGMs are not uncommon and are undoubtedly due to one of three factors or a combination of two or all of them; namely, the inability of the host stroma to provide sufficient tissue to form a strong PGM, uneven alignment of host and graft Descemet's membranes, and irregular trauma to the marginal endothelium of the graft.

Concerning the aetiology of RCMs, the results of the present and previous experiments indicate, in agreement with Brown and Kitano (1966), that any situation deleterious to the corneal endothelium in association with a rupture in Descemet's and the deep stromal layers is to be considered as an underlying cause. It remains only to suggest that circumstances sometimes must supervene which are equally deleterious to RGM parent cells, even though the three postulated conditions for membrane formation (Sherrard and Rycroft, $1967 \mathrm{~b}$ ) are satisfied, with the result that RGMs will not necessarily form in all cases where they might be expected on clinical and histological criteria.

\section{Summary}

Fragments of corneal stroma explanted into the anterior chamber generated neofibrous tissue, providing they were free of endothelium, in the same way that stroma exposed in posterior corneal wounds generates retrocorneal membranes. Stromal explants fixed against the retrocorneal surface became endothelialized fairly rapidly and little neofibrosis occurred, while explants separated from the endothelium by silicone rubber membranes 
grew extensively. The endothelium of the average homograft is probably often inferior to that of the host, with the result that endothelialization of the host/graft junction may occur from the host side before it commences from the graft. This may explain the characteristic retrograft position of the post-graft membrane.

I am indebted to Prof. G. J. Cunningham for his guidance and advice throughout this work, to the late Peter V. Rycroft for many stimulating discussions and ideas, and to the late Sir Benjamin Rycroft for his interest and $\varrho$ encouragement. I extend my thanks to Mr. Arthur Barron for the photographs, and to Mrs. E. Rodgers and ڤ્心 Miss A. Bullock for technical assistance.

\section{References}

BINDER, R. F., and BINDER, H. F. (I957) A.M.A. Arch. Ophthal., 57, I I BRown, S. I., and Kitano, s. (1966) Arch. Ophthal. (Chicago), 75, 518

Chi, н. H., teng, c. C., and katzin, н. м. (i96o) Amer. 7. Ophthal., 49, 693

dohlman, c. H., brown, s. I., and martola, e. l. (ig67) Trans. Amer. Acad. Ophthal. Otolaryng., 71, $\bigodot^{\circ}$ 85 I

KURz, G. H., and D'Amico, R. A. (1968) Amer. 7. Ophthal., 66, I84

Mills, N. L., and Donn, A. (1960) Arch. Ophthal. (Chicago), 64, 443

morton, P. L., ORMSBy, H. L., and basu, P. к. (1958) Amer. 7. Ophthal., 46, pt 2, p. 62

Peña-CARrillo, J., and POlACK, F. M. (1964) Arch. Ophthal. (Chicago), 72, 81 I

RobBins, J. E., CAPella, J. A., and kaufman, H. E. (1965) Ibid., 73, 242

RYCROFT, P. V. (I965) Trans. ophthal. Soc. L.K., 85, 3 I 7

SHERRARD, E. s. (1966) Vet. Rec., 78, 253

- and RYCROFT, P. v. (1967a) Brit. 7. Ophthal., 51, 379

- ( $1967 \mathrm{~b}) \quad$ Ibid., 51, 387

тномаs, с. I. (I955) "The Cornea." Thomas, Springfield, Ill.

WERB, A., (1962) Int. Ophthal. Clin., 2, 77 I 